\title{
A reanalysis of the theory of interferometer experiment demonstrating that Michelson's analysis contains an error, including the boat model analysis, so readmitting the ether presence
}

\author{
Ioan Has ${ }^{1}$, Simona Miclaus ${ }^{2}$, Aurelian Has ${ }^{3}$ \\ ${ }^{1}$ Land Forces Academy Sibiu, st. Rapsodiei nr. 6B, bl. Sof 1, sc. B, ap. 9, 240240, Rm. Valcea, Romania \\ ${ }^{2}$ Land Forces Academy, st. Revolutiei. 3-5, 550170 Sibiu, Romania \\ ${ }^{3}$ University “C. Brancoveanu”, st. N. Balcescu nr.39, 240200 Rm. Valcea, Romania
}

\section{Email addresses:}

hasavo@yahoo.com (I. Has), simo.miclaus@gmail.com (S. Miclaus), hasaurelian@yahoo.com (A. Has)

\section{To cite this article:}

Ioan Has, Simona Miclaus, Aurelian Has. A Reanalysis of the Theory of Interferometer Experiment Demonstrating that Michelson's Analysis Contains an Error, Including the Boat Model Analysis, so Readmitting the Ether Presence. Optics. Vol. 3, No. 4, 2014 , pp. 24-32.

doi: $10.11648 /$ j.optics.20140304.11

\begin{abstract}
Theoretical basis of Michelson interferometer experiment is reanalyzed. Initially we reanalyzed the illustrative model, represented by a boat traversing a river which revealed that the correct transversal trajectory is not an isosceles triangle, but a right triangle. Also in the interferometer, the transversal double light path, considering the ether presence and classical theories of light, we found to be too a right triangle instead isosceles one considered by Michelson. But this new path necessitates zero time difference, for which fringes displacements are not expected, unlike Michelson's analysis. So we found that the light's double travel times don't depend on the interferometer arms directions and so the expected by Michelson fringes displacements, must not appear. The above findings are applicable to other similar experiments with ray double travel, including light, lasers, masers, $\gamma$-rays, etc. We demonstrated that Michelson's experiment correct interpretation does not confirm the relativity of the light speed. Consequently, Michelson's experiment should not be considered negative concerning the presence of ether which must remain the bearer of the $E-M$ phenomena. Consequently, the Special Relativity Theory (SRT) must be reconsidered, based on Lorentz electromagnetic theory from 1895 and the quanta entanglement and E-M field can be physically explained by the ether presence.
\end{abstract}

Keywords: General Physics, Geometrical Optics, Light Interference, Michelson Experiment, Ether, SRT

\section{Classical Conditions of the Experiment}

Here we reanalyzed the correctness of the paths and of the traveling times, of the two orthogonal rays from Michelson's interferometer experiment, resulted by splitting a light ray and re-encountering them finally.

Michelson's experiment, which was first carried out in 1881 and repeated with Morley in 1887 [1], is based on Maxwell's suggestion and aimed to find the speed of the ether wind by "determination of light speeds by measuring the times it needs to travel in two opposite directions a known distance." [2]. We will prove that the Michelson's interferometer does not fulfill this suggestion.
The layout of the rotating Michelson's interferometer device is presented in the same layout in position I (Pos I), in the majority of works on the subject, while position II (Pos. II), with the arms rotated by $90^{\circ}$, wasn't analyzed but only was supposed to yield identical results to Pos. I. [3-7]. In fact, the two arms formed by the light source $(\mathrm{S})$, the observation telescope $(\mathrm{L})$ and the two mirrors $\left(\mathrm{A}_{1}, \mathrm{~A}_{2}\right)$ are disposed at $90^{\circ}$ to one another, and the semitransparent plate $\mathrm{P}$ is disposed at $45^{\circ}$, as shown in Fig. 1. [1, 2].

We note that in Michelson's analysis the geometrical optics theory was admitted as valid, including a hypothetical ether, which must be present also in our analysis.

In reassessments of Michelson's experiment made over the past few decades, no objection has been expressed about correctness of its theoretical basis starting from light paths, 
putting in discussion other aspects, most regarding the experiment development [8,9]. And most of these approaches are developed in the SRT assumptions, and not in the classical conditions and analysis as this work does.

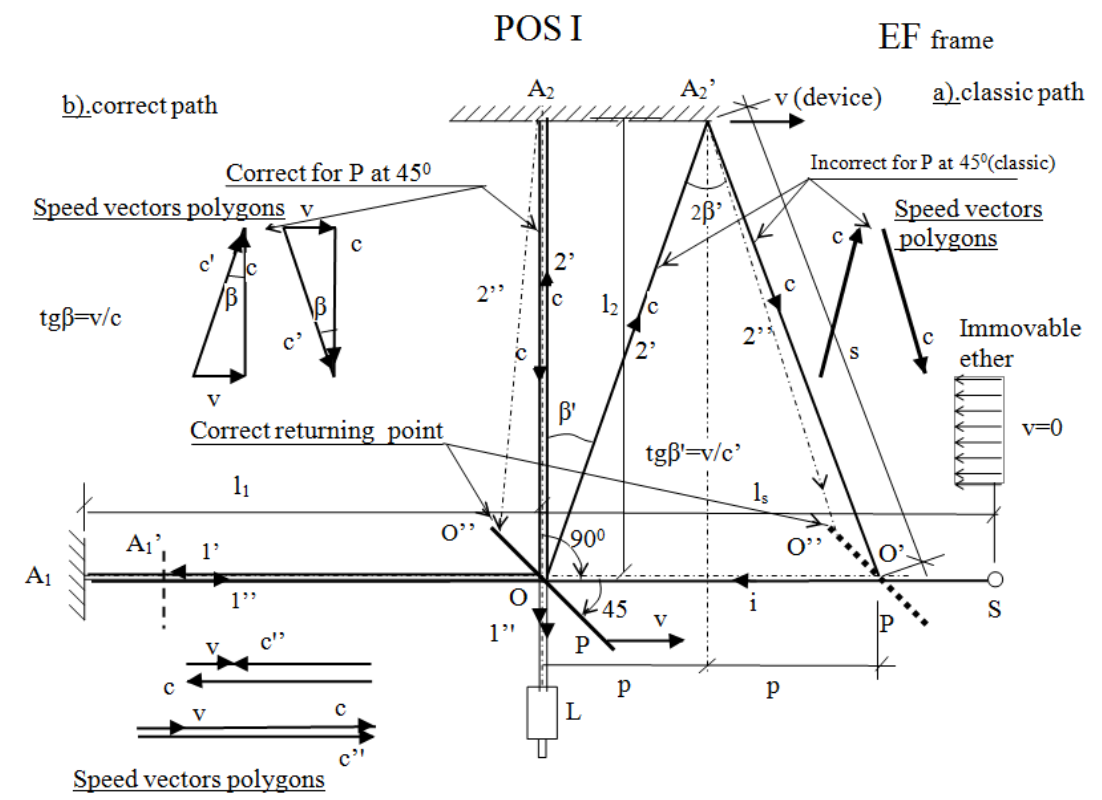

Fig 1. Classical arrangement of Michelson's interferometer. a). Classic paths in EF frame with movable device. b). Corrected paths in EF frame.

For correct analysis of the light path, the reference frame must be clear established. The frame must be attached to the considered immovable part of the experiment, which may be the device (DF frame), or the ether (EF frame), by choice. In this article the reference frame, will be indicated in each figure by (DF, EF), and by the zero speed $\left(v_{i}=0\right)$ of the immovable part. Both frames have the same value, but the frame (DF) is preferred in this paper for calculations.

However, in almost all precedent works on the subject, including Michelson's analysis, the EF frame (immovable ether) were tacitly (no such indication given) adopted [2, 3] in which frame the correct light path construction is more complicated and ambiguous and maybe has contributed to some errors.

Under these conditions, for the transversal ray $2^{\prime}-2^{\prime \prime}$ an isosceles path $O A_{2}^{\prime} O^{\prime}$ was admitted by Michelson as in Fig. 1a in EF frame, path which will prove to be wrong. The longitudinal path $1^{\prime}-1^{\prime \prime}$ was admitted to be $O A_{1}^{\prime} O^{\prime}$ and it was considered that the two rays return to the same point $O^{\prime}$, and so the interference principal condition was theoretically ensured. So, the two times $t_{1}$ and $t_{2}$ the light needs to travel the two device arms in Pos. I (Fig.1a) was obtained as follows [2]:

For the ray $1^{\prime} 1^{\prime \prime}$ with the path $O A_{1}^{\prime} O^{\prime}$ the time $t_{1}$ is:

$$
t_{1}=t_{1}^{\prime}+t_{1}^{\prime \prime}=\frac{l_{1}}{c+v}+\frac{l_{1}}{c-v}=\frac{2 l_{1}}{c} \frac{1}{1-v^{2} / c^{2}}=\frac{2 l_{1}}{c} \frac{1}{\alpha^{2}}
$$

For the ray $2^{\prime}-2^{\prime \prime}$ with the isosceles path $\mathrm{OA}_{2}^{\prime} \mathrm{O}^{\prime}$ (EF frame) the time $t_{2}$ is (with $\alpha=\frac{1}{\sqrt{1-v^{2} / c^{2}}}$ )

$$
t_{2}=t_{2}^{\prime}+t_{2}^{\prime \prime}=\frac{2 l_{2}}{c} \frac{1}{\sqrt{1-v^{2} / c^{2}}}=\frac{2 l_{2}}{c} \frac{1}{\alpha}
$$

Considering $l_{1}=l_{2}$ for Pos. I, the time difference is:

$$
\left(t_{1}-t_{2}\right)_{\mathrm{I}}=\frac{l}{c} \frac{v^{2}}{c^{2}}
$$

By rotating the device counterclockwise by $90^{\circ}$ to Pos. II, it was supposed by Michelson and in all precedent classical works, that the two rays 1 and $2^{\prime \prime}$ return to the same point $o^{\prime}$ ensuring the interference. Thus the total time difference due to the device rotation with $90^{\circ}$ was obtained by Michelson as follows:

$$
\Delta t=\left(t_{1}-t_{2}\right)_{\mathrm{I}}-\left(t_{1}-t_{2}\right)_{\mathrm{II}}=\frac{2 l}{c} \frac{v^{2}}{c^{2}}
$$

This time difference should create a fringe translation $\Delta \mathrm{N}$ which in the experiment from 1887 should have been $\Delta N=0.37$ fringe. However, the value measured by Michelson $[10,11]$ was much smaller than that calculated and was neglected, whilst the experiment was considered "negative" concerning the presence of ether conducting at SRT's birth.

Here we remark that in Michelson's analyses, rays 1 and 2 were tacitly supposed to start their travel at the same initial moment, and that they would both arrive at the point $\mathrm{O}$ where they interfere, after two different times $t_{1}$ and $t_{2}$.

However, the interference at the $\mathrm{O}$ point is produced only if the following main conditions are satisfied:

i). The two light rays must meet at a certain point in space, $\mathrm{O}$;

ii). The two rays must arrive at $\mathrm{O}$ both at a certain moment, $t$

iii). The two rays must be the most coherent;

We see that in Michelson's analysis condition i). may be fulfilled by a correct geometrical optics construction of the light paths.

In order to fulfill condition ii)., we see that in the case of two different times, with $t_{1}>t_{2}$ it is necessary for the ray 2 to 
start from the source $\mathrm{S}$ after the ray 1 starts, with a time difference of $\Delta t=t_{1}-t_{2}$.

The condition iii). may be fulfilled conditionally. In interferometer, the rays $1^{\prime \prime}$ and $2^{\prime \prime}$ will have maximum coherence when they correspond to a minimum $\Delta t$, or better

to $\Delta t=0$.

\section{Traversing River Boat Model Reanalysis}

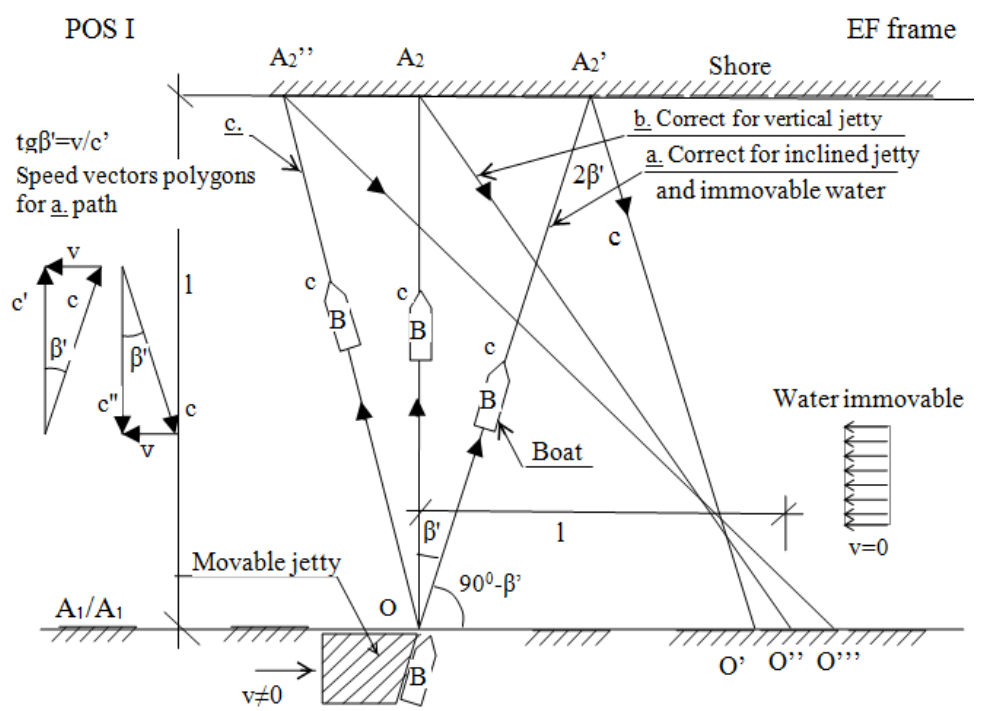

Fig 2. The boat model for the interferometer. Paths in EF frame with movable jetty and immovable water.

Initially, the classic interferometer model of a boat or of a swimmer traversing a river, as it is presented in Fig.2, will be reanalyzed more closely. This model, with small adaptations in many works and physics books, was considered to be similar to the light paths in the interferometer placed in the ether wind $[11,12]$. It was admitted in these works, that the real transversal path of the boat, in EF frame, is the isosceles triangle $\mathrm{OA}_{2}^{\prime} \mathrm{O}^{\prime}$ (" $a$ " in Fig.2). In these works the boat's start direction (given by jetty direction) was admitted to be inclined at $90^{\circ}$ as in the device (Fig.1 a).

But in Fig.2, based on velocity polygons in EF frame, we observe that the correct jetty launching direction must have an inclination of $90^{\circ}-\beta^{\prime}$, in order to produce the initial boat velocity $c$, inclined also by $90^{\circ}-\beta^{\prime}$ (where $\operatorname{tg} \beta^{\prime}=v / c^{\prime}$, whith $c^{\prime}=\sqrt{c^{2}-v^{2}}$ or $\left.\beta^{\prime} \cong v / c^{\prime}\right)$.

Otherwise, with the jetty and boat inclined at $90^{\circ}$, with water immovable, the boat will have the departure path $\mathrm{OA}_{2}$ and will arrive at the opposite point $\mathrm{A}_{2}$ by "b" path [13].

But a boat starting with an inclination of $90^{\circ}-\beta^{\prime}$ depending on the unknown $v$, has an unfair advantage, an inadmissible situation.

It is noteworthy that Rosser [11] admits an upstream path inclination of his swimmer, but without considering and drawing the correctly inclined start direction, which is a stolen start in sport, and therefore inadmissible.

The two times, $t_{1}$ and $t_{2}$ for the boat paths $O A_{1}^{\prime} O^{\prime}$ and $\mathrm{OA}_{2}^{\prime} \mathrm{O}^{\prime}$ ("a" from Fig.2) resulted in the classic works with the same expressions as for the light in interferometer, given by s. (1) and (2).

In Fig. 2 a possible correct path "b" in EF frame, is also presented for the jetty inclined at $90^{\circ}$. Consequently, in this situation the boat will start off, in the vertical direction (the path $\mathrm{OA}_{2}$ ). In this case, in order for the boat to return to the point of appointment with the movable jetty, the returning path must be $\mathrm{A}_{2} \mathrm{O}$ " ("directed" by its captain, observing the jetty speed). The resulting path in EF frame is a right triangle $\mathrm{OA}_{2} \mathrm{O}^{\prime \prime}$.

But in Fig.2, there are a multitude of possible traversing paths like "c" ( $O A_{2}^{\prime \prime} O$ " $)$, with $A_{2}^{\prime \prime}$ situated anywhere on the opposite shore, while the longitudinal path $O A_{1} O^{\prime}$ is unique [13].

But in a "fair contest" of boats or of swimmers along the two directions, the transversal departure direction must be at 900 , because the captain or the swimmer does not know the water velocity or the $\beta$ ' inclination, in advance. However, from opposite shore they can observe the jetty movement and can anticipate the appointment point.

The precedent observations will help us to reanalyze the Michelson's analysis of experiment. In Sec. 5 we will demonstrate that for the right triangle $\mathrm{OA}_{2} \mathrm{O}$ " (Fig. 2) the time $t_{2}$ is equal with $t_{1}$ or $\Delta t=0$, and consequently the boat or the swimmer arrives at finish in the same time, regardless of the traveling direction, result which must be valid also for the light paths in an interferometer.

\section{The Reanalysis of Michelson's Analysis of the Experiment}

Now we return to Fig.1a in which the classic light path $O A_{2}{ }^{\prime} O^{\prime}$ is represented in EF frame, and we will compare this path to the similar boat path $\mathrm{OA}_{2}^{\prime} \mathrm{O}^{\prime}$ from Fig. 2 .

We notice here the inadvertence between the position of $\mathrm{P}$ plate inclined at $45^{\circ}$ (Fig.1) and the position of the boat and jetty, correctly inclined at $90^{\circ}-\beta^{\prime}$ (Fig.2). However, both divergent positions of the $\mathrm{P}$ plate and of the jetty are the basis 
for the same isosceles paths $O_{A_{2}^{\prime}} O^{\prime}$ for the EF frame in Fig.1a and respectively $\mathrm{O}_{2}{ }^{\prime} \mathrm{O}^{\prime}$ in Fig.2, in classical analyses.

Now we can observe that there appears an error in the departure path $O A_{2}^{\prime}$ of the classical ray path from Fig.1a, which does not correspond correctly to the $45^{\circ}$ inclined position of the plate P in Fig. 1 as we try in Sec. 4.

Or for avoiding the error, in geometrical optics one must incline conveniently $\mathrm{P}$ plate.

Here in Fig. 1 in EF frame, the interferometer is considered movable with $\mathrm{v}$ speed to right, while the ether is immovable, and for this reasons the correct reflection path must be at 900 represented by $\mathrm{OA}_{2}$ path in Fig. $1 \mathrm{~b}$ for EF frame.

From speed vectors polygons, the complete correct transversal path in Fig.1b will be $O A_{2} O$, but interrupted at level of O" point due to P plate movement. We see here the difficulty of representing graphically this path in EF frame.

With these correct paths from Fig.1b, the condition i). (Sec. 1) for the light interference is not correct fulfilled, because the $O$ " point doesn't coincide with the $O$ point, and the interference is not assured theoretically [14-16].

It must be remarked that Kittel et. al. [7] noticed that the two rays $1^{\prime \prime}$ and $2^{\prime \prime}$ do not return to the same point but surprisingly concluded that this does not affect the analysis and calculations, including the interference conditions, which conclusion can not be true.

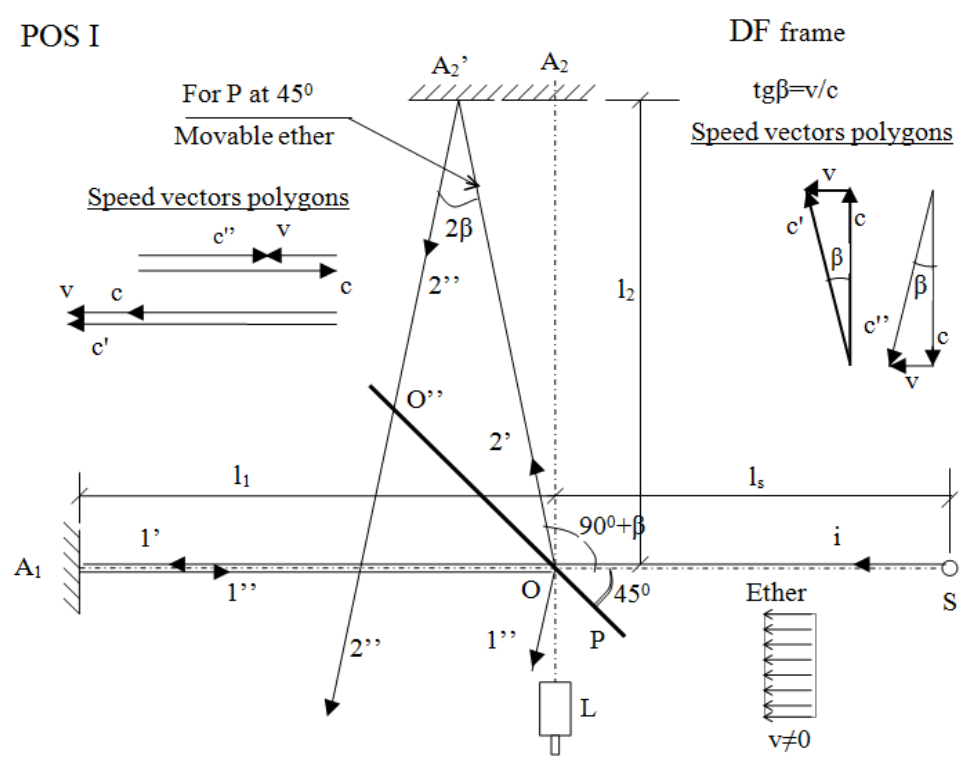

Fig 3. Corrected light paths in Pos. I. with the device in classical arrangement, in DF frame with immovable device.

The same device layout in Pos. I in EF frame, can be better analyzed considering the DF frame as in Fig.3, where the correct paths $O A_{2}^{\prime} O^{\prime}$ and $O A_{1} O$ were built by geometrical optics. For this purpose, velocity polygons presented for each direction were used, and here again the points $\mathrm{O}$ and $O^{\prime \prime}$ are different and the condition i). of the interference is not assured.

Here we can make the general observation that in all the situations when the ether is considered movable, DF frame, with a speed $v$, one must work with $c^{\prime}$ light speed, which is the vector sum of the $\mathrm{c}$ and $\mathrm{v}$ vectors. A reflected ray direction must be obtained, taking into account only the reflection of the light speed $c$ vector (real speed in ether, and not of the resultant $c^{\prime}$ vector) with its real incident direction

The direction of the light speed resultant vector c' after an effective reflection must be obtained by composing the reflected $\mathrm{c}$ vector with the $\mathrm{v}$ vector of the ether velocity.

Using this rule, the correct paths $O A_{2}^{\prime} O{ }^{\prime \prime}$ and $O A_{1} O$ were obtained, utilizing the velocity polygons, in Fig.3. Here, we observe clearly that the condition $\mathrm{i}$ ). of interference for rays $1^{\prime \prime}$ and $2^{\prime \prime}$ is not assured because $\mathrm{O}$ and $O^{\prime \prime}$ points on $\mathrm{P}$ plate do not coincide.

We mention here that light paths in the interferometer, but rotated by $90^{\circ}$ to Pos. II, can be easily constructed also in DF frame [17]. And also in Pos. II, the condition i). of interference of the two light paths will be not assured.

We now conclude that with a classical arrangement in Pos. I of the interferometer, in EF frame with $\mathrm{P}$ plate inclined at $45^{\circ}$ (Fig.3), and considering the effects of an ether wind $\mathrm{v}$ like Michelson, the transversal path $\mathrm{OA}_{2}^{\prime} \mathrm{O}^{\prime}$ from Fig.1a as isosceles triangle, as admitted by Michelson, is incorrect.

The corrected path $\mathrm{OA}_{2} \mathrm{O}^{\prime \prime}$ for the same device arrangement presented in Fig. $1 \mathrm{~b}$ as overlapped paths (or $\mathrm{OA}_{2}^{\prime} \mathrm{O}^{\prime \prime}$ from Fig.3 in DF frame), also does not assure theoretically the i). condition of the interference from Sec.1.

\section{A Possible Device Arrangement of $P$ Plate for Obtaining the Classical Light Path}

We now remark that positioning the $\mathrm{P}$ plate inclined at $\left(45^{\circ}+\beta^{\prime} / 2\right)$ angle [14-16], as in Fig.4, appears to be a possible method for assuring the concordance of the interferometer arrangement and the Michelson classical ray path, $O A_{2}{ }^{\prime} O^{\prime}$ from Fig. 1a. in EF frame, and Pos.I.

In this new arrangement of the device from Fig.4a in EF frame, the ray $2^{\prime}-2^{\prime \prime}$ will have the correct optical geometrical path as the classical path $O_{A_{2}^{\prime}} O^{\prime}$ as isosceles 
triangle.

Also in this new arrangement of the device from Fig. $4 \mathrm{~b}$ but in DF frame, the ray 2'-2" will have the correct optical geometrical path $\mathrm{OA}_{2} \mathrm{O}$, as superposed paths.

While the $1^{\prime}-1^{\prime \prime}$ ray, Fig. $4 \mathrm{a}, 4 \mathrm{~b}$, has the same previous paths $O A_{1}^{\prime} O^{\prime}$ or $\mathrm{OA}_{1} \mathrm{O}$, in $\mathrm{EF}$ frame, respectively in $\mathrm{DF}$ frame.

In this way the two rays $1^{\prime}-1^{\prime \prime}$ and $2^{\prime}-2^{\prime \prime}$ return to the same

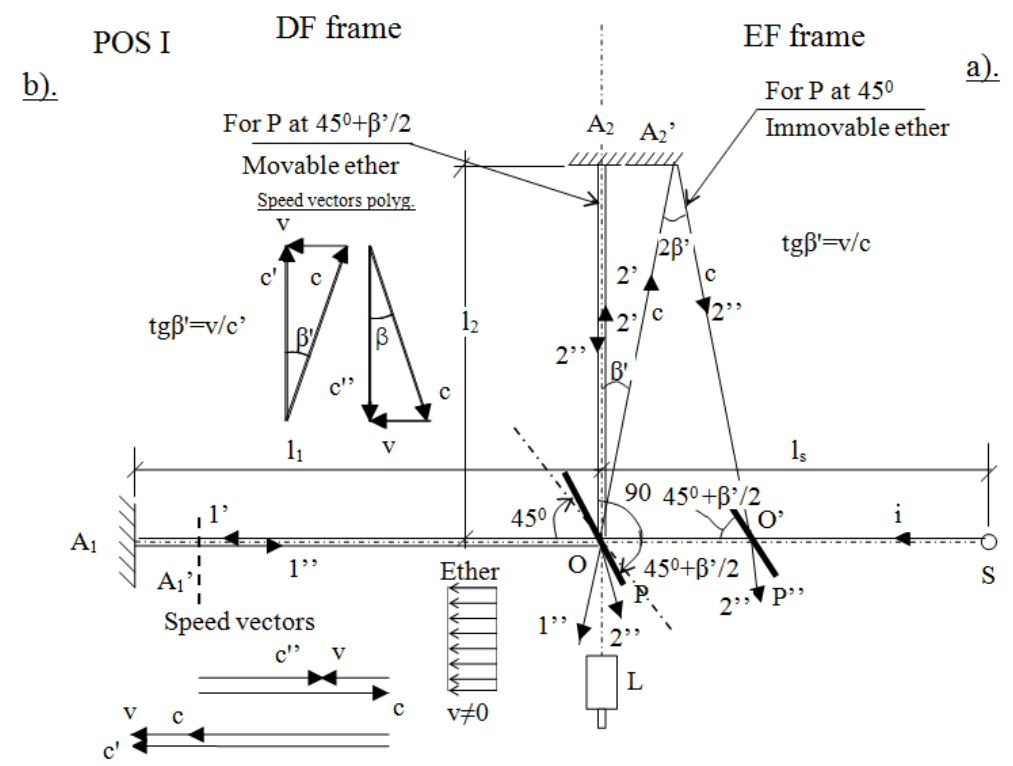

Fig 4. Corrected light paths in Pos. I. with the device having the P plate inclined at $45^{0}+\beta^{\prime} / 2$. a). In EF frame. b). In DF frame including speed vectors polygons.

However, it was necessary to also verify the interference in Pos. II for the same device arrangement [P at $\left.\left(45^{\circ}+\beta^{\prime} / 2\right)\right]$, operation which was done previously [18]. In the case of Pos. II it resulted from [18] that the interference condition i). of the two rays is not ensured theoretically with $\mathrm{P}$ at $\left(45^{\circ}+\beta{ }^{\prime} / 2\right)$. The same negative results will be obtained after two other rotations of $90^{\circ}$ to Pos III and IV, as can be easily demonstrated [18].

But now we must remark that a ray 2' (Fig.4) which starts as in Michelson's experiment with an inclination $90^{\circ}+\beta^{\prime}$ in EF frame, depending on the ether wind speed $v$, unknown prior to it, would have to be an "intelligent ray" which is not the case. Or the P plate must be inclined by a mechanism with a variable angle $\beta$ ' with the device rotation in each moment, but this possibility with $\mathrm{P}$ plate inclined at $\left(45^{\circ}+\beta^{\prime} / 2\right)$ for assuring the concordance between interferometer arrangement and ray (2') is not realistic, and must be abandoned.

\section{A Method to Establish an Alternative Light Path Ensuring the Interference in Classic Device Arrangement}

point $O^{\prime}$ in $\mathrm{EF}$ frame (respectively $\mathrm{O}$ in $\mathrm{DF}$ frame), the first condition i). of interference being fulfilled as Michelson theoretically considered for Pos. I, but here in Fig.4 P plate is inclined at $\left(45^{\circ}+\beta^{\prime} / 2\right)$.

But the condition iii). is not best fulfilled, because the two times are unequal and the coherence will be less good than that of the two rays having the same times. 


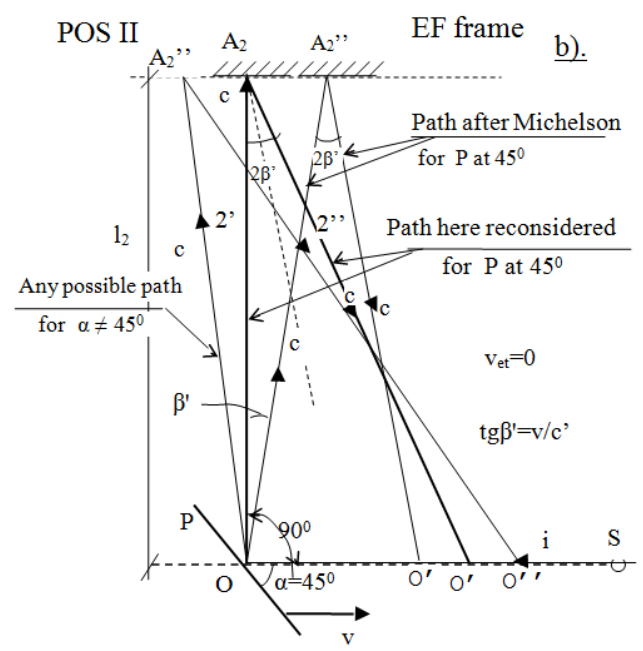

Fig 5. Reconsidered light paths in Pos. I with the ray 2" returned to O point, as Huygens/Fresnel reemitted ray. , a). In DF frame including speed vectors polygons. b). In EF frame.

In order to find a method of ensuring the interference theoretically in the classical device arrangement and conditions, we can suppose that the transverse returning ray (2" in Fig.3) can arrive at $\mathrm{O}$ or very closely to point $\mathrm{O}$ for assuring the interference, as happened in the experiment. This case of Pos. I is presented in Fig.5a, in DF frame.

For this purpose it is possible to apply the HuygensFresnel undulatory light theory, in which all points of a surface including mirrors, constitute new secondary light sources that spreads rays in any direction. This hypothesis applied to the $A_{2}$ mirror surface touched by a light wave 2', can give some backward reemitted rays 2" arriving from point $A_{2}^{\prime}$ also at $\mathrm{O}$ point.

Another possibility for this purpose is to incline $A_{2}$ mirror with $\beta$ ' angle in Pos. I, but in Pos. II the effect is contrary as is easily to demonstrate [17], and this solution must be too abandoned.

This hypothesis and phenomenon of secondary source must be correct for small $\beta$ ' angles as those corresponding to earth-ether speed. Maybe at greater speeds the interference will be negative affected.

Moreover, the same phenomenon produces in the case of laser devices for measuring distances, when the returning ray is reflected by any surface which is not a mirror, and is received by a lateral lens.

In this way the transversal ray 2 " returns to point $\mathrm{O}$ by path $\mathrm{A}_{2}$ 'O because $c^{\prime} \| c^{\prime \prime}$, as results from speed polygons in Fig.5a.

The ray 1" also returns to point $\mathrm{O}$, theoretically ensuring in this way all the tree conditions of interference from Sec.1.

In Fig.5b in EF frame, one can see that the path of the ray

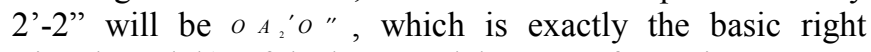
triangle path $b$ ). of the boat model [15-18] from Fig.2.

This hypothesis of applying Huygens-Fresnel theory and phenomenon of secondary source of light to the interferometer, must be correct also because in boat model, the boat path was the same, a right triangle, and this model path can be proved in reality.

Now, we will calculate the travel time $t_{2}$ necessary for the light to perform the transversal path $O A_{2}{ }^{\prime} O^{\prime \prime}$ from Fig. $5 \mathrm{~b}$. in EF frame, from where we can write:

$$
t_{2}=t_{2}^{\prime}+t_{2}^{\prime \prime} ; t_{2}^{\prime}=\frac{l_{2}}{c}=\frac{O A_{2}}{c} ; t_{2}^{\prime \prime}=\frac{A_{2}^{\prime} O^{\prime \prime}}{c} ; O O^{\prime \prime}=v\left(t_{2}^{\prime}+t_{2}^{\prime \prime}\right)=v t_{2}
$$

From the right triangle $\mathrm{OA}_{2} \mathrm{O}^{\prime \prime}$ we have:

$$
\left(A_{2}^{\prime} O^{\prime \prime}\right)^{2}=\left(O A_{2}^{\prime}\right)^{2}+\left(O O^{\prime \prime}\right)^{2}
$$

Introducing (5) into (6) we obtain:

$$
\begin{aligned}
& \left(t_{2}^{\prime \prime} c\right)^{2}=\left(t_{2}^{\prime} c\right)^{2}+\left(\begin{array}{ll}
t_{2} \\
2
\end{array}\right)^{2} \\
& \left(t_{2}^{\prime \prime} c\right)^{2}=\left(t_{2}^{\prime} c\right)^{2}+v^{2}\left[\left(t_{2}^{\prime}\right)^{2}+2 t_{2}^{\prime} t_{2}^{\prime \prime}+\left(t_{2}^{\prime \prime}\right)^{2}\right] \\
& \left(t_{2}^{\prime \prime}\right)^{2}\left(c^{2}-v^{2}\right)-2 v^{2} t_{2}^{\prime} t_{2}^{\prime \prime}-\left(t_{2}^{\prime}\right)^{2}\left(c^{2}+v^{2}\right)=0
\end{aligned}
$$

Solving the above second-degree equation in $t_{2}^{\prime \prime}$ gives the following result:

$$
t_{2}^{\prime \prime}=\frac{2 v^{2} t_{2}^{\prime}+\sqrt{4 v^{4}\left(t_{2}^{\prime}\right)^{2}+4\left(c^{2}-v^{2}\right)\left(c^{2}+v^{2}\right)\left(t_{2}^{\prime}\right)^{2}}}{2\left(c^{2}-v^{2}\right)}=\frac{v^{2} t_{2}^{\prime}+t_{2}^{\prime} \sqrt{v^{4}+\left(c^{2}-v^{2}\right)\left(c^{2}+v^{2}\right)}}{c^{2}-v^{2}}
$$

Since the solution $t_{2}^{\prime \prime}=0$ will be banal, we choose the + sign in front of the square root in (10). Combining (5) and (10) we obtain:

$$
t_{2}=t_{2}^{\prime}+t_{2}^{\prime \prime}=\frac{l_{2}}{c}+\frac{\frac{v^{2} l_{2}}{c}+\frac{l_{2}}{c} \sqrt{v^{4}+c^{4}-v^{4}}}{c^{2}-v^{2}}=\frac{l_{2}}{c}+\frac{\frac{v^{2} l_{2}}{c}+\frac{l_{2} c^{2}}{c}}{c^{2}-v^{2}}=\frac{l_{2} c^{2}-l_{2} v^{2}+v^{2} l_{2}+c^{2} l_{2}}{c\left(c^{2}-v^{2}\right)}
$$

And finally from (11) we get:

$$
t_{2}=\frac{2 l_{2} c^{2}}{c\left(c^{2}-v^{2}\right)}=\frac{2 l_{2}}{c} \frac{1}{1-v^{2} / c^{2}}=\frac{2 l_{2}}{c} \frac{1}{\alpha^{2}}
$$

We observe that in (12) was obtained for $t_{2}$ an expression identical to those found for $t_{1}$ in (1).

And the expression for $t_{1}$ from (1) remains, being correct, although that simple classic calculation is inconsequent concerning reference frames.

The correct $t_{1}$ calculation in DF frame is more complicated as was shown in [18] and it gives the same result as in (1).

When the device arms are equal, from (1) and (12), $\Delta t$ results:

$$
\Delta t=t_{1}-t_{2}=0
$$

Thus, in this case of Pos. I in Fig.5a or b, we can say that no time difference exists between the light travel times $t_{1}$ and $\mathrm{t}_{2}$ in the too directions.

As result, also the ii). and iii). conditions of interference from Sec. 1 will be fulfilled because the rays 1" and 2 " derive from the same initial $i$ ray, assuring the best coherence because of the minimum (zero in fact) time difference.

Hence, the other rays reflected in others points except $\mathrm{A}_{2}$ point, arriving at $\mathrm{O}$ point, including the isosceles ray $O A_{2}^{\prime \prime} \mathrm{O}^{\prime}$ from Fig. 5b), will be stumped by $\mathrm{OA}_{2}^{\prime} \mathrm{O}$ " ray because of their greater time difference, and their worse coherence, and the visible interference fringes will be that produced by rays 1 " and $\mathrm{OA}_{2}^{\prime} \mathrm{O}^{\prime \prime}$ (Fig. 5b), or by $1^{\prime \prime}$ and $2^{\prime \prime}$ rays (Fig. 5a). 
Here we note that even the isosceles path of Michelson analysis, which may be obtained too by applying HuygensFresnel theory in O point for 2' ray (Fig.1, Fig.3, Fig.5b) will give the same significant $\Delta \mathrm{t} \neq 0$ and such a path will be stumped by the right angle path with smaller $\Delta \mathrm{t}$ or even $\Delta \mathrm{t}=0$, which will give a better coherence.

The best coherence corresponds to the path which gives real energy transfer between the source and the visible fringe. The energy transfer can take place in a point only once. In this case the energy transfer for fringes take place by two rays from two paths and it will be most favorable in conditions of the best coherence, which is firstly influenced by $\Delta \mathrm{t}$ minim, even zero.

The result given by (13) from Fig. 5a). in DF frame for Pos I, will be true also for Pos. II, III and IV of the similar device arrangement and ray paths, due to axial partial symmetry. These situations may be easily verified in Fig. 6 for Pos. II, where the source arm length $1_{\mathrm{s}}$ was neglected. In Fig. 6 we obtained the same transversal and longitudinal paths for 1" and 2" rays as in Fig.5a, but in new arms positions and with inversed senses.

With our precedent hypotheses regarding the transversal light paths as right triangle in Michelson interferometer and considering the results of the above analytical demonstration, we get that the fringe displacements are theoretically null because of $\Delta t=0$ in any rotated position of the interferometer. And consequently, in such tip of interference experiments is not justified to expect the large values of fringe displacement predicted theoretically by Michelson.

We observe that the paths as an isosceles triangle in EF frame from Fig.1, and Fig.5b proposed by Michelson represent extreme paths for the $t_{2}$ time (a minimum), and a maximum value for $\Delta \mathrm{t}$. For these paths, because $\Delta t \neq 0$, interference conditions i)., ii), iii), from Sec. 1 will not be well fulfilled and these rays will be stomped by other rays arriving at the same point $\mathrm{O}$, with the minimum $\Delta \mathrm{t}$ and better coherence.

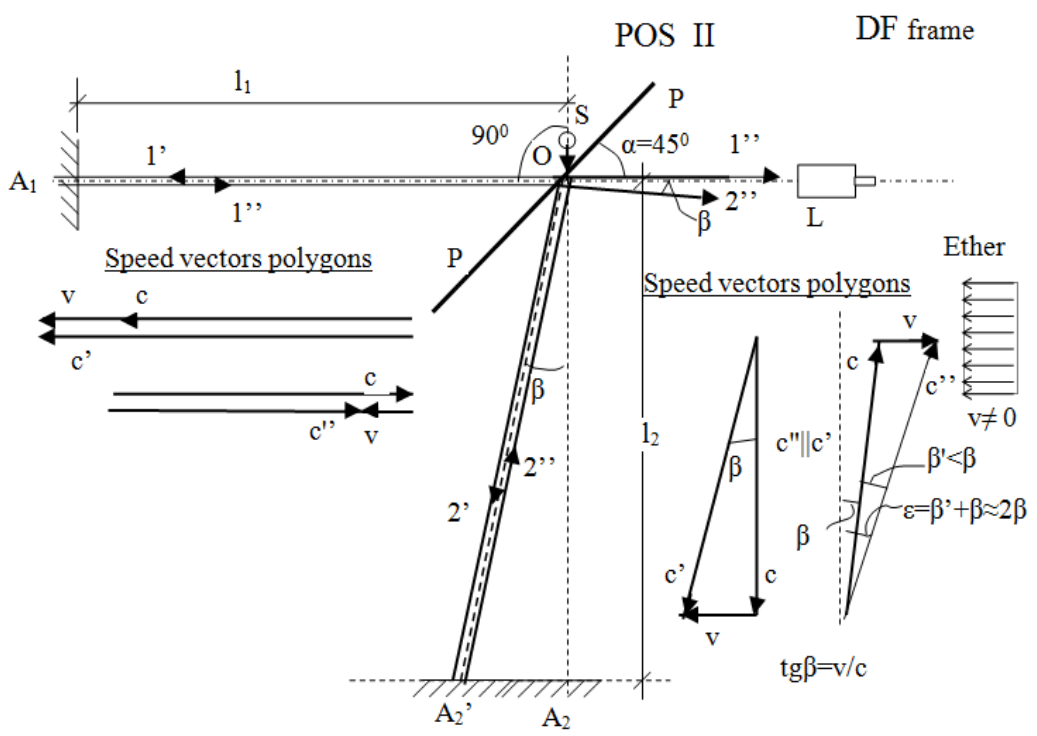

Fig 6. Reconsidered light paths in DF frame including speed vectors polygons, in Pos. II with the ray 2 " returned to O point, as Huygens/Fresnel reemitted ray

Such extreme paths with maximum $\Delta \mathrm{t}$ are unlikely to stay at the origin of the visible interference fringes which necessitate the real energy transfer by best coherence, as was shown above.

Some similar results presented Mark [19] reanalyzing Michelson analysis, concluding that the value of $\Delta \mathrm{t} \neq 0$ obtained by Michelson is not correctly deduced and it must be $\Delta \mathfrak{t}=0$. But Mark's similar theoretical experiment with two balls/light rays, traveling in a moving cubic cabin, differs significantly from our case by completely dragged atmosphere.

\section{Consideration about Observed Fringe Displacements in Interferometer Experiments}

Some little fringe displacements may appear by rotating the device between Pos. I and Pos. IV because the ether wind influence is real. This wind can influence the $t_{1}$ and $t_{2}$ times by at least the four perturbing factors: ray direction, finite width of light ray, source distance, v speed [14].

Some paths near the right triangle in EF frame in Fig. 5b, or near the $\mathrm{O}_{2} \mathrm{O}$ path in Fig. 5a in DF frame giving some non null $\Delta \mathrm{t}$, must be at the origin of such observed little fringes displacements, in most interference experiments.

One can also obtain such small values of $\Delta t$, if the fringes are regarded not as result of a single ray, but as a component of a multitude of rays constituting a light beam with finite width.

In such conditions some small values can be obtained for $\Delta \mathrm{t}$, which are not exactly zero but small enough to be observed as in Michelson's and in other similar experiments, which are probably third order effects of the ether wind.

Under above conditions with the device rotated in Pos. I., II., III and IV., the interference may be only approximately stationary (very small displacements), as in Michelson's and other experiments. 
Our precedent reanalysis and above observations, are in good agreement with the detailed results of Michelson's experiments, registering some small fringe displacements.

Michelson, [10] have reported small fringe displacements in its well known graphic for 24 hours. Our measurement [17] on this graphic, indicated some clear fringe displacements of approximately $0,02 \lambda$, which represent approx. $5 \%$ of the expected theoretical movement $(0,37 \lambda)$, which is not at all negligible, as was considered till now.

Other accurate experiments made later with more improved interferometers have constantly shown however, that in fact some small time differences $\Delta t$ appeared, reaching $1 / 3$ of the classical theoretical time difference [12].

Also Miller [20] had reported fringe displacements in all of his numerous experiments, which reached even. $20 \%$ of the expected theoretical displacement, amount which can be diminished by actual error analysis, but which can't be considered zero.

These observed small fringe displacements may constitute an argument for the ether wind presence, in the light of our above results.

\section{Conclusions and Consequences}

In this article we have demonstrated that the transverse light path in the rotating interferometer, similar to the boat path traversing a river, admitted by Michelson as isosceles triangle in EF frame, is not consistent with a correct analysis when performed under classic conditions of geometrical optics.

We have shown, that the transversal ray path which is an isosceles triangle in EF frame in Michelson's analysis, must start with an inclination $\beta^{\prime}$ depending on the ether wind speed, unknown to it before leaving the source. Such a ray behavior, implying to be an "intelligent ray", is not possible, except the case of applying Huygens-Fresnel theory, which was never invoked by Michelson and its followers, or critics.

We were successful in finding, based on geometrical optics and on undulatory light theory, that a simple transversal ray must start at $90^{\circ}$ and perform a right triangle path in EF frame, in order to return in the same point with the longitudinal ray, and resulting equal times and the same moment of returning.

Some other analyses of Michelson experiment was made in last period [ex.: 21, 22, 23] but they are not based on, nor discuss our hypothesis of right triangle path of light in the interferometer.

For this transversal right triangle path we calculated a time, longer than that obtained by Michelson, but equal with the time from longitudinal path. In these circumstances are fulfilled also the best interference conditions.

Even if the Michelson isosceles triangle may be constructed by applying Huygens-Fresnel theory at point $\mathrm{O}$, it will give a bad coherence and it will be stumped by the right triangle ray. This right triangle ray will give the best coherence and will correspond to the real energy transport which yields the visible fringes.
And because the time difference $\Delta t$ results zero, no fringe displacement must appear by rotating the device despite Michelson's anticipations.

So the interferometer problem with the supposed ether presence is far from being as simple as it was considered by Michelson in his theoretical analysis, which was incomplete and consequently incorrect

The Michelson's experiment, which is based only on the existence of time difference $\Delta t$ for a double light paths, and not on the measurements of simple times for two simple orthogonal paths of light travel, do not correspond to Maxwell's recommendation for ether wind speed measurements.

And consequently, only other type of experiment based on the simple travel time, are suited for this purpose of establishing the relative movement speed of the earth in ether.

Because of other small dependencies of light paths and travel times, on the ether wind direction in such a complex optical device, as presented in Sec. 3, 4, 5, 6, 7, it is logical to admit that an absolute stationary interference fringe pattern is practically improbable to persist. So small $\Delta \mathrm{t}$ ' will result, compared with $\Delta \mathrm{t}$ calculated by Michelson, which can be attributed to ether wind as tertiary effect.

But the interference and small fringe displacement created by $\Delta \mathrm{t}$ ' may subsist theoretically at relative small ether speed around the earth. Maybe at greater ether-device speed the interference will be more affected.

In fact, only an approximate stationary fringe figure was obtained in Michelson's experiments in the classic hypothesis of the ether wind influence, when small displacements was also obtained in all similar experiments including Miller's experiment, for which our above results, confirm as being justified his prolonged efforts.

Presently our reinterpretation of Michelson's analysis does not allow to consider his experiment as a confirmation of relativity of the light speed.

And based on our precedent findings we conclude that Michelson's experiment must be no longer considered as negative concerning the presence of the ether wind.

Under these conditions, the longitudinal arm contraction proposed by Fitzgerald is not necessary, and consequently nor is necessary the SRT proposed by Einstein on the basis of Michelson experiment analysis. Results that Fitzgerald, Lorentz and Einstein were mislead by Michelson's wrong analysis of its interferometer experiment. But Lorentz electromagnetic theory from 1895, actualized, based on an immobile ether, may substitute SRT.

The ether presence can also physically explain, the quanta entanglements of microparticles including bosons, regarded as local stationary oscillations in the ether components.

And the ether presence can constitute the physical support of the electro-magnetic (E-M) field or of other fields, assuring the universal interaction transmitted with light sped $c$, which is a property of the ether and not of the emission source.

Similar experiments [24, 25] including light, microwaves, masers, lasers, $\gamma$ rays, etc., which are based on the time 
difference $\Delta t$ calculation and measurements (for the two orthogonal or inclined double paths) given by relationships similar to those in equations (1) and (2), which do not correspond to the correct Maxwell suggestion for measuring the ether wind speed, may also be reanalyzed in light of the above findings.

In order to successfully put into evidence the ether wind speed, the correct experiments must actually fulfill Maxwell's suggestions by measuring the simple times for two orthogonal simple paths, and such experiments would be possible in the future, including more precise observations of Jupiter satellites eclipses.

A similar situation was happened in antiquity when Ptolemy proposed his functional geocentric planet system, based on apparent planet observed movements, as Einstein did in 1905 for his SRT based on apparent and wrong light path in Michelson experiment, as we demonstrated above. Both these theories can describe the reality only until a limited detail or precision, but finally they can be proved to be wrong, because the planets in reality rotates around the sun as Copernicus demonstrated, and because the light must have a propagation support, a kind of ether, for whose properties discovery, we must conduct our future efforts.

\section{Acknowledgements}

The first author acknowledges the initial advice on the subject and encouragement of his late professor $\mathrm{N}$. Barbulescu. He is sincerely grateful to prof. P. Mazilu from TUCB Bucharest for his rigorous lessons of rationality. He is also indebted to Gen. prof. N. Stoinea, to Gen. prof. dr. G. Barsan from LFA Sibiu, to prof. N. Vasiliu, to prof. D. Siposan from MTA Bucharest, to Ms. Laurel Sullivan from NHS, USA, to Ms. prof. Madalina Popescu, to Mr. Michael Tate and to Miss Veronica Has, for their continuous support. For intermediate developments of this theme the authors appreciate the support of the MEC (Grant under Contract no.05-D11-54/2005).

\section{References}

[1] A. A. Michelson, E. W. Morley, On the relative motion of the earth and the luminiferous ether. Am. J. Sci., 34, 333-345, (1887)

[2] N. Barbulescu, Einstein's relativity physical basis (in Romanian. Ed. St.\& Enc., Bucharest, 1979 p.59,).

[3] M. Born, Die Relativtatstheorie Einsteins, (in Romanian, Ed. Stiintifica, Bucharest, 1969).

[4] J. Boutigny, Optique, (Vuibert, Paris, 1983).

[5] R..P. Feynman, Lectures on physics, Vol. I, (Addison-Wesley, Reading, Massachusetts, 1964.)

[6] D. Sivoukhine, Cours de physique generale, Tom. IV, (Ed. Mir, Moscou, 1984)
[7] C. Kittel, W. D Knight, M. A Ruderman, Mechanics, Berkley physics course. Vol. I. (in Romanian), (Ed. Didactica \& Pedagogica Bucharest p.335, 1981).

[8] S. J. Prokhovnik, The logic of special relativity, (Cambridge Univ. Press, 1967)

[9] E. F. Taylor, J. A. Wheeler, A la decouverte de l'espace-temps, (Dunod, Paris, 1970).

[10] A. A. Michelson, Studies in optics, (Phoenix Science Series, Chicago, 1968).

[11] W..G.V. Rosser, An introduction to the theory of relativity. (Butterworths, London, 1964)

[12] B. Jaffe, Albert Michelson and light speed ( in Romanian, Ed. Stiintifica, Bucharest, 1974).

[13] I. Has, "A reconsideration of the illustrative example of Michelson's experiment " (in Romanian), Rev. Fizica \& Chimie. XXXI/11-12, (Bucharest) 14-18 (1996)

[14] I. Has, A new interpretation of Michelson's experiment, (in Romanian). Rev. Fizica \& Chimie. XXXII/ 6-7-8, (Bucharest). $1-5(1997)$

[15] I. Has, The analysis of an alternative light path in Michelson's experiment. Rom. Rep. Phys. 52, 775-789 (2000)

[16] I. Has, Considerations about motion phenomena starting from a model for relativity theory. In Volume the $\mathrm{IV}^{\text {th }}$ Congress of "S. Odobleja" Academy, (Ed. Europa Nova, Bucharest, 1997). 51-65.

[17] I. Has, S. Miclaus, A. Has, An alternative light path analysis in Michelson's experiment. Physics Essays. 23, 248-257 (2010)

[18] I. Has, A. Has, Light paths analysis in classic interferometer conditions. Balkan Phys. Lett. (proc. Suppl. BPL-4) 67-70 (2000)

[19] H. H. Mark, Speed of light, the vanished points of reference. Optics. 3(2), 12 -14 (2014)

[20] D. C. Miller. The ether-drift experiment and the determination of the absolute motion of the earth. Rev. Mod. Phys., 5(3), 203-242 (1933).

[21] R. C. de Miranda Filho. First order effects in the MichelsonMorley experiment. Physics Essays., 15(4), 422-438 (2002).

[22] H. A. Munera, Michelson-Morley experiment revisited: systematic errors, consistency among different experiments, and compatibility with absolute space. Apeiron 5(1-2), 37-53 (1998).

[23] H. A. Munera, The effect of solar motion upon the fringeshift in a Michelson-Morley interferometer a la Miller. Anales de la Fondation Louis de Broglie. 27(3), 463-484 (2002).

[24] T. S. Jaseja, A. Javan, J. Murray, C. Townes, Test of special relativity or of the isotropy of space by use of infrared masers. Phys. Rev. 133, A1221 (1964)

[25] R. J. Kennedy, E. M Thorndike, Experimental establishment of the relativity of time. Phys. Rev., 42(3), 300-418 (1932). 